IRA-International Journal of Management \& Social Sciences

ISSN 2455-2267; Vol.04, Issue 01 (2016)

Institute of Research Advances

http://research-advances.org/index.php/RAJMSS

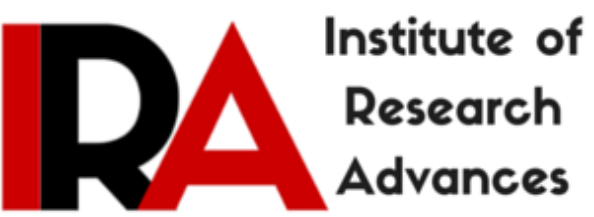

\title{
A Study of Sustainable Disclosure Practices of Indian Corporates
}

\author{
Shilpa S. Motwani \\ Assistant Professor \\ Gujarat Commerce College \\ Ellisbridge, Ahmedabad, India. \\ Dr. Hemal B. Pandya ${ }^{2}$ \\ Professor \\ S. D. School of Commerce \\ Gujarat University, Ahmedabad, India.
}

DOI: http://dx.doi.org/10.21013/jmss.v4.n1.p13

\section{How to cite this paper:}

Motwani, S., \& Pandya, H. (2016). A Study of Sustainable Disclosure Practices of Indian Corporates. IRA-International Journal of Management \& Social Sciences (ISSN 2455-2267), 4(1). doi:http://dx.doi.org/10.21013/jmss.v4.n1.p13

(C) Institute of Research Advances

\section{(cc) BY-NC}

This works is licensed under a Creative Commons Attribution-Non Commercial 4.0 International License subject to proper citation to the publication source of the work.

Disclaimer: The scholarly papers as reviewed and published by the Institute of Research Advances (IRA) are the views and opinions of their respective authors and are not the views or opinions of the IRA. The IRA disclaims of any harm or loss caused due to the published content to any party. 


\begin{abstract}
The corporate governing bodies have worked towards governance, socially responsibility over the last couple of decades. They are now emphasising sustainability reporting. The focus has now shifted from financial to the non-financial aspects of reporting. Sustainability reporting also termed as Triple-bottom-line reporting, which means the company has now to report on non financial aspects like environmental, social, governance along with the economic aspect. This is evident by the release of the National voluntary guidelines on Social, Environmental and Economic responsibility of business (NVG-SEE) by Ministry of Corporate Affairs, India in 2011; followed by SEBI mandating Clause 55 of the listing agreement with stock exchange in India in 2012; making Business responsibility reporting (BRR) compulsory for the top 100 listed companies (by market capitalisation). These non financial aspects have been well incorporated by the Global Reporting Initiative (GRI); a non-profit organisation providing sustainability guidance.

This paper is an attempt to study the comparative analysis of sustainability disclosure practices of Indian companies using GRI based index. The sustainability disclosures have been studies companywise and Industry-wise. The study concludes that Indian companies have recognized the importance of sustainability reporting and the major companies are reporting on it. However, Indian companies do not give much consideration to their industries characteristics while disclosing information in their sustainability reports.
\end{abstract}

Key Words: Sustainability Reporting disclosure, Global Reporting Initiative

\title{
1. Introduction
}

The corporate governing bodies have worked towards governance, socially responsibility over the last couple of decades. They are now emphasising sustainability reporting. The focus has now shifted from financial to the non-financial aspects of reporting. Sustainability reporting also termed as Triplebottom-line reporting, which means the company has now to report on non financial aspects like environmental, social, governance along with the economic aspect. This is evident by the release of the National voluntary guidelines on Social, Environmental and Economic responsibility of business (NVG-SEE) by Ministry of Corporate Affairs, India in 2011; followed by SEBI mandating Clause 55 of the listing agreement with stock exchange in India in 2012; making Business responsibility reporting (BRR) compulsory for the top 100 listed companies (by market capitalisation). These non financial aspects have been well incorporated by the Global Reporting Initiative (GRI); a non-profit organisation providing sustainability guidance.

The increasing mandations towards sustainability have made the companies and its stakeholders much aware, and started looking towards the sustainable aspects. 'Sustainability', which more simply means -fulfilling the needs of the present generation, without compromising the needs of the future generations.

This paper is an attempt to study the comparative analysis of sustainability disclosure practices of Indian companies using GRI based index.

\section{Literature review}

(Bhatia \& Tuli, 2014); tried to study and assess the extent and level of sustainability reporting in India. They concluded saying that Indian companies are recognising the importance of sustainability reporting. Their results showed an optimistic picture regarding sustainability.

(Godha \& Jain, 2015); reviewed sustainability reporting and its benefits for the Indian Companies. They examined the development in the Indian regulatory environment for sustainability reporting along with finding out trend, application level and status of the sustainability reporting practice of Indian entities as per the GRI reporting framework. Their findings reveal that the development of the 
sustainability reporting is maturing in India. Indian companies are increasingly realizing that they have much to lose by not following sustainability reporting.

(Goyal, 2014); studied industry-wise corporate sustainability reporting practices among Indian companies by selecting companies on BSE 500 index from different five sectors. He studied the level of corporate sustainability disclosures in India, and also the most frequent disclosed items. $\mathrm{He}$ concluded that sustainability reporting is gaining importance with the passage of time. Due to lack of a general framework for reporting, he found lot of variations in the disclosures by companies and industries.

(Mitra, 2012); studied the state of sustainability disclosures of Indian companies and found that many Indian companies are reporting complete information relevant to the reporting organisation.

(Kumar, 2014); examined and compared the sustainability reporting practices in the selected petroleum companies in the NSE nifty index companies and FORTUNE 50 companies basis of GRI. He found the NSE Nifty sample companies' disclosure practices to be very good than the Global Fortune 50 sample companies.

(Daizy \& Das, 2014); made comparative analysis of two famous sustainability reporting framework: GRI (Global Reporting Initiative) and DJSI (Dow Jones Sustainability Index); and found that GRI is considered very much acceptable framework for sustainability reporting as compared to DJSI.

(Bhalla \& Bansal, 2014); analysed the growth of sustainability reporting in India, with regard to only the economic aspect of reporting. They found that Indian companies have started actively participating in reporting the sustainability issues using GRI.

\section{Research Questions:}

The present study attempts to answer the following questions.

1. What are the sustainability disclosure practices of Indian Companies?

2. What is the extent of sustainability disclosures by Indian Companies?

\section{Objectives}

On the basis of the above questions and the literature review, the following are the major objectives of the present study:

$>$ To study the sustainability disclosure practices of selected Indian companies

$>$ To know whether the disclosure practices of selected Indian companies among industries differ significantly from each other or not.

\section{Hypothesis}

With reference to the objectives of the study and to know whether the disclosure practices of selected Indian companies among industries differ significantly from each other or not; the following Hypothesis were framed,

H0: There is no significant difference in the inter industry disclosure scores.

H1: There is a significant difference in the inter industry disclosure scores.

\section{Research Methodology}

A. Period of the study: the sustainable disclosure practices of the selected companies have been studied for the period of two years i.e. 2014-15 and 2013-14. The reason for choosing these two years is that we found maximum number of companies disclosing with the same GRI version in these two years.

\section{B. Selection of Sample:}

The samples were selected on the criteria that the company should be listed on NSE, with large market capitalization and should exclusively report on sustainability on the basis of GRI versions viz. (G4 and G 3.1) 
The final sample size stood as follows:

\begin{tabular}{|c|c|c|c|}
\hline \multicolumn{2}{|c|}{ G 4 } & \multicolumn{2}{c|}{ G 3.1 } \\
\hline $2014-15$ & $2013-14$ & $2014-15$ & $2013-14$ \\
\hline 23 & 17 & 11 & 24 \\
\hline
\end{tabular}

\section{Source of Data:}

The research is purely based on the secondary data of publically listed companies. The data was retrieved from the sustainability reports of the companies, and the sustainability reports were obtained from the official website of the selected companies.

\section{Research Method:}

This study aims to study the sustainability disclosure practices of Indian companies; hence content analysis has been used for the purpose of analysis. An index based on GRI version G3.1 and G4 was used to analyse the extent of disclosures. The reason to choose the GRI based index is that it is a more comprehensive and universally accepted. The following is the detail classification of the index based on GRI version G3.1 and G4:

Table 1: Classification of Index of Disclosure into Categories as per GRI G 3.1

\begin{tabular}{|c|l|c|}
\hline Sr. No & \multicolumn{1}{|c|}{ Categories } & No. of Items \\
\hline 1 & Strategy and analysis & 2 \\
\hline 2 & Organisational profile & 10 \\
\hline 3 & Report parameters & 13 \\
\hline 4 & Governance, commitment, \& engagement & 17 \\
\hline 5 & Management approach & 6 \\
\hline 6 & Performance indicators & 9 \\
\hline 6.1 & Economic & 30 \\
\hline 6.2 & Environmental & 15 \\
\hline 6.3 & Social & 11 \\
\hline 6.3 .1 & Labour practices \& decent work & 10 \\
\hline 6.3 .2 & Human rights & 9 \\
\hline 6.3 .3 & Society & $\mathbf{1 3 2}$ \\
\hline 6.3 .4 & Product responsibility \\
\hline \multicolumn{2}{|c}{ Total } & \\
\hline
\end{tabular}

Table 2: Classification of Index of Disclosure into Categories as per GRI G 4

\begin{tabular}{|c|l|c|}
\hline Sr. No & \multicolumn{1}{|c|}{ Categories } & No. of Items \\
\hline 1 & Strategy and analysis & 2 \\
\hline 2 & Organisational profile & 11 \\
\hline 3 & Commitments to external initiatives & 3 \\
\hline 4 & Identified Material Aspects and Boundaries & 7 \\
\hline 5 & Stakeholder Engagement & 4 \\
\hline 6 & Report Profile & 6 \\
\hline 7 & Governance & 22 \\
\hline 8 & Ethics and Integrity & 3 \\
\hline 9 & Performance indicators & 10 \\
\hline 9.1 & Economic & 34 \\
\hline 9.2 & Environmental & 16 \\
\hline 9.3 & Social & 12 \\
\hline 9.3 .1 & Employment & 11 \\
\hline 9.3 .2 & Human rights & 9 \\
\hline 9.3 .3 & Society $\quad$ Total \\
\hline 9.3 .4 & Product responsibility \\
\hline & $\quad$
\end{tabular}


The details of the above tables indicate the maximum disclosure. This was compared with the disclosures made by each company. The scoring was done as follows:

\begin{tabular}{|c|c|}
\hline Nature & Score \\
\hline Fully reported & 2 \\
\hline Partially reported & 1 \\
\hline Not reported & 0 \\
\hline Not material & 0 \\
\hline Not applicable & Excluded \\
\hline
\end{tabular}

The final scores were converted into percentage and ranks were allotted to the companies. The company with maximum percentage of disclosure was allotted first rank and so forth.

Further, industry wise disclosures were analysed using one way ANOVA to see if the disclosure practices of companies among industries differ significantly from each other or not. For this purpose the disclosures of companies chosen was for the year 2014-15 and reporting as per GRI G4. The reason for analysing the same was that for the other years and version of GRI we did not found any mean differences; and also this data being the latest will reflect the present scenario.

\section{Results and Discussions}

The sustainability disclosure practices of Indian companies have been summarized as follows:

Where,

F- $\quad$ Fully Disclosed items

P- Partially Disclosed items

NO- Not disclosed items

NA- Not Applicable

Total- Total Items to be disclosed

S- $\quad$ Score obtained

AS- Applicable Score

Per- Percentage Score

Table 3: Company-wise disclosure for the year 2014-15 as per GRI G4

\begin{tabular}{|c|c|c|c|c|c|c|c|c|c|c|c|}
\hline \multicolumn{12}{|c|}{ Company-Wise Disclosure } \\
\hline \multicolumn{12}{|c|}{$\begin{array}{c}\text { G4 } \\
2014-15\end{array}$} \\
\hline $\begin{array}{l}\text { Sr. } \\
\text { No. }\end{array}$ & COMPANY & $\mathbf{F}$ & $\mathbf{P}$ & NO & NA & Total & $\mathbf{S}$ & $\mathbf{A S}$ & S/AS & Per & Rank \\
\hline 1 & AXIS BANK & 108 & 0 & 42 & 0 & 150 & 216 & 300 & 0.72 & 72.00 & 11 \\
\hline 2 & $\mathrm{BP}$ & 135 & 0 & 11 & 4 & 150 & 270 & 292 & 0.92 & 92.47 & 3 \\
\hline 3 & GAIL & 129 & 0 & 20 & 1 & 150 & 258 & 298 & 0.87 & 86.58 & 5 \\
\hline 4 & HDFC BANK & 91 & 0 & 52 & 7 & 150 & 182 & 286 & 0.64 & 63.64 & 16 \\
\hline 5 & HINDALCO & 134 & 9 & 7 & 0 & 150 & 277 & 300 & 0.92 & 92.33 & 4 \\
\hline 6 & HONDA & 117 & 0 & 27 & 6 & 150 & 234 & 288 & 0.81 & 81.25 & 8 \\
\hline 7 & $\mathrm{HP}$ & 59 & 0 & 91 & 0 & 150 & 118 & 300 & 0.39 & 39.33 & 20 \\
\hline 8 & INFOSYS & 100 & 9 & 37 & 4 & 150 & 209 & 292 & 0.72 & 71.58 & 12 \\
\hline 9 & IOCL & 148 & 0 & 1 & 1 & 150 & 296 & 298 & 0.99 & 99.33 & 1 \\
\hline 10 & ITC & 124 & 1 & 20 & 5 & 150 & 249 & 290 & 0.86 & 85.86 & 6 \\
\hline 11 & $\begin{array}{l}\text { Jindal Steel \& } \\
\text { Power Ltd } \\
\text { JSPL }\end{array}$ & 52 & 0 & 98 & 0 & 150 & 104 & 300 & 0.35 & 34.67 & 21 \\
\hline 12 & JK CEMENT & 52 & 0 & 98 & 0 & 150 & 104 & 300 & 0.35 & 34.67 & 22 \\
\hline 13 & L\&T & 116 & 0 & 34 & 0 & 150 & 232 & 300 & 0.77 & 77.33 & 9 \\
\hline
\end{tabular}


IRA-International Journal of Management \& Social Sciences

\begin{tabular}{|c|l|c|c|c|c|c|c|c|c|c|c|}
\hline 14 & $\begin{array}{l}\text { MAHINDRA } \\
\text { finance }\end{array}$ & 68 & 0 & 79 & 3 & 150 & 136 & 294 & 0.46 & 46.26 & 19 \\
\hline 15 & NEROLAC & 128 & 0 & 22 & 0 & 150 & 256 & 300 & 0.85 & 85.33 & 7 \\
\hline 16 & OIL INDIA & 72 & 6 & 72 & 0 & 150 & 150 & 300 & 0.50 & 50.00 & 18 \\
\hline 17 & SAIL & 51 & 0 & 99 & 0 & 150 & 102 & 300 & 0.34 & 34.00 & 23 \\
\hline 18 & $\begin{array}{l}\text { TATA } \\
\text { CHEMICALS }\end{array}$ & 98 & 0 & 52 & 0 & 150 & 196 & 300 & 0.65 & 65.33 & 14 \\
\hline 19 & $\begin{array}{l}\text { TATA } \\
\text { MOTORS }\end{array}$ & 92 & 0 & 57 & 1 & 150 & 184 & 298 & 0.62 & 61.74 & 17 \\
\hline 20 & $\begin{array}{l}\text { TATA } \\
\text { POWER }\end{array}$ & 148 & 0 & 2 & 0 & 150 & 296 & 300 & 0.99 & 98.67 & 2 \\
\hline 21 & TATA STEEL & 109 & 0 & 40 & 1 & 150 & 218 & 298 & 0.73 & 73.15 & 10 \\
\hline 22 & Tech Mahindra & 98 & 0 & 52 & 0 & 150 & 196 & 300 & 0.65 & 65.33 & 15 \\
\hline 23 & YES BANK & 101 & 0 & 49 & 0 & 150 & 202 & 300 & 0.67 & 67.33 & 13 \\
\hline
\end{tabular}

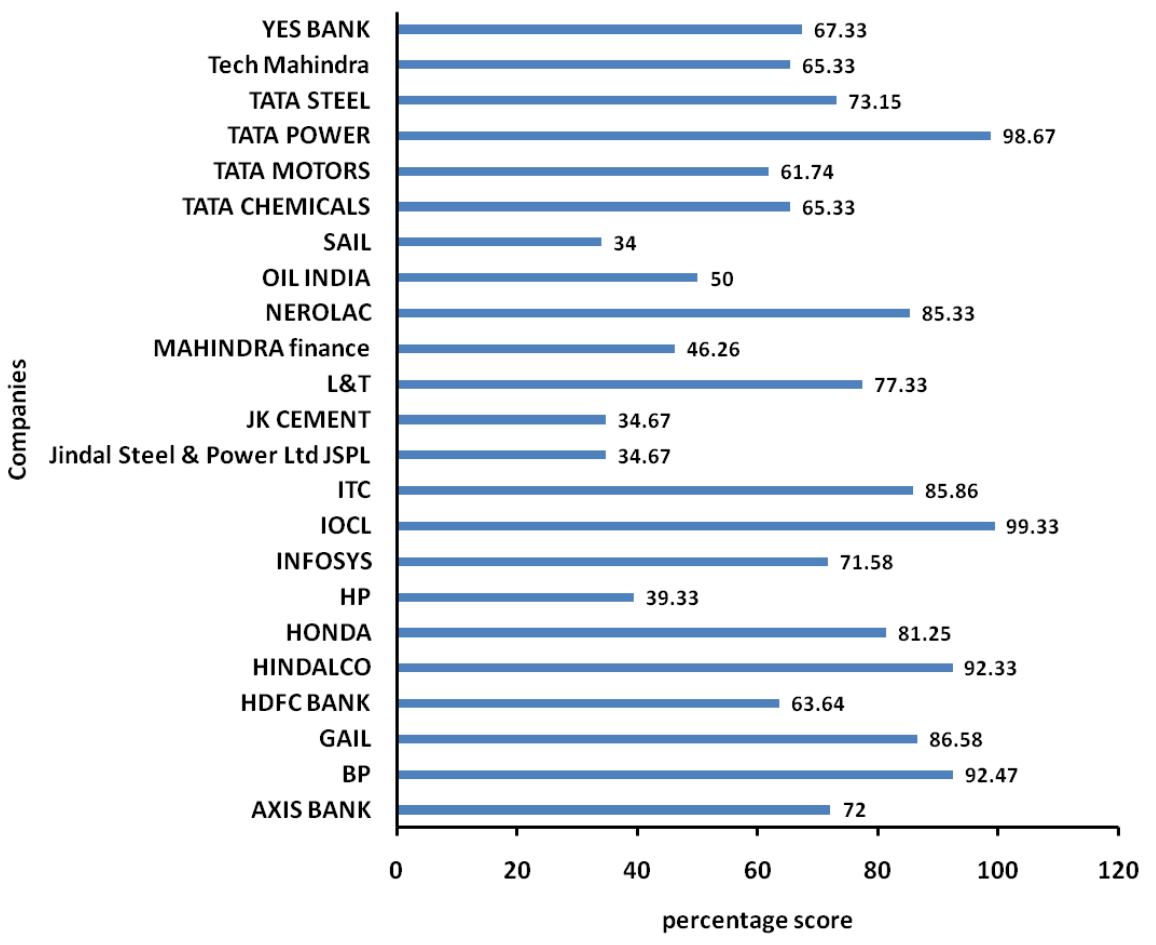

Figure 1: Company-wise disclosure for the year 2014-15 as per GRI G4

Table 4: Company-wise disclosure for the year 2013-14 as per GRI G4

\begin{tabular}{|c|l|c|c|c|c|c|c|c|c|c|c|}
\hline \multicolumn{10}{|c|}{$\mathbf{2 0 1 3 - 1 4}$} \\
\hline Sr. No. & \multicolumn{1}{|c|}{ COMPANY } & F & P & No & NA & Total & S & AS & S/AS & Per & Rank \\
\hline 1 & ACC & 84 & 1 & 62 & 3 & 150 & 169 & 294 & 0.57 & 57.48 & 12 \\
\hline 2 & AMBUJA CEMENT & 140 & 6 & 4 & 0 & 150 & 286 & 300 & 0.95 & 95.33 & 2 \\
\hline 3 & BHEL & 138 & 0 & 11 & 1 & 150 & 276 & 298 & 0.93 & 92.62 & 3 \\
\hline 4 & BP & 135 & 0 & 11 & 4 & 150 & 270 & 292 & 0.92 & 92.47 & 4 \\
\hline 5 & COLGATE & 53 & 0 & 97 & 0 & 150 & 106 & 300 & 0.35 & 35.33 & 15 \\
\hline 6 & Godrej \& Boyce Manufacturing & 40 & 0 & 110 & 0 & 150 & 80 & 300 & 0.27 & 26.67 & 17 \\
\hline 7 & INFOSYS & 106 & 5 & 34 & 5 & 150 & 217 & 290 & 0.75 & 74.83 & 8 \\
\hline
\end{tabular}


IRA-International Journal of Management \& Social Sciences

\begin{tabular}{|c|l|c|c|c|c|c|c|c|c|c|c|}
\hline 8 & IOCL & 144 & 0 & 6 & 0 & 150 & 288 & 300 & 0.96 & 96.00 & 1 \\
\hline 9 & ITC & 119 & 0 & 26 & 5 & 150 & 238 & 290 & 0.82 & 82.07 & 6 \\
\hline 10 & Jindal Steel \& Power Ltd JSPL & 51 & 0 & 99 & 0 & 150 & 102 & 300 & 0.34 & 34.00 & 16 \\
\hline 11 & MARUTI SUZUKI & 86 & 0 & 64 & 0 & 150 & 172 & 300 & 0.57 & 57.33 & 13 \\
\hline 12 & NEROLAC & 134 & 0 & 16 & 0 & 150 & 268 & 300 & 0.89 & 89.33 & 5 \\
\hline 13 & NOVARTIS & 122 & 0 & 28 & 0 & 150 & 244 & 300 & 0.81 & 81.33 & 7 \\
\hline 14 & OIL INDIA & 52 & 12 & 86 & 0 & 150 & 116 & 300 & 0.39 & 38.67 & 14 \\
\hline 15 & SHREE CEMENT & 106 & 0 & 43 & 1 & 150 & 212 & 298 & 0.71 & 71.14 & 9 \\
\hline 16 & TATA CHEMICALS & 99 & 0 & 51 & 0 & 150 & 198 & 300 & 0.66 & 66.00 & 10 \\
\hline 17 & YES BANK & 87 & 0 & 63 & 0 & 150 & 174 & 300 & 0.58 & 58.00 & 11 \\
\hline
\end{tabular}

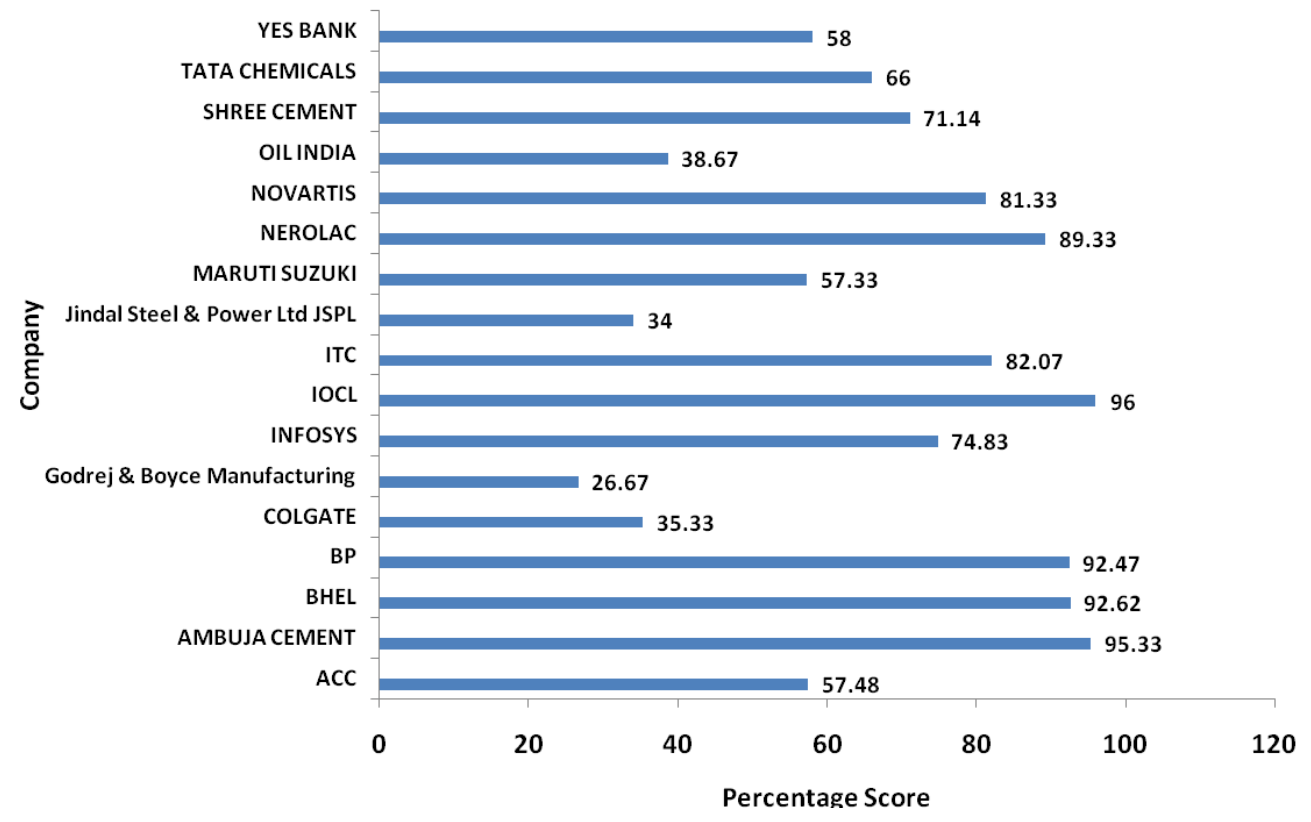

Figure 2: Company-wise disclosure for the year 2013-14 as per GRI G4

Table 5: Company-wise disclosure for the year 2014-15 as per GRI G3.1

\begin{tabular}{|c|l|c|c|c|c|c|c|c|c|c|c|}
\hline \multicolumn{10}{|c|}{ G3.1 } \\
\hline Sr. No. & \multicolumn{1}{|c|}{ COMPANY } & F & P & No & NA & Total & S & AS & S/AS & Per & Rank \\
\hline 1 & AIRTEL & 88 & 0 & 19 & 25 & 132 & 176 & 214 & 0.82 & 82.24 & 8 \\
\hline 2 & CAIRAN INDIA & 98 & 11 & 23 & 0 & 132 & 207 & 264 & 0.78 & 78.41 & 10 \\
\hline 3 & COAL INDIA & 94 & 0 & 38 & 0 & 132 & 188 & 264 & 0.71 & 71.21 & 11 \\
\hline 4 & ESSAR shipping & 95 & 14 & 20 & 3 & 132 & 204 & 258 & 0.79 & 79.07 & 9 \\
\hline 5 & HCL technologies & 117 & 3 & 1 & 11 & 132 & 237 & 242 & 0.98 & 97.93 & 2 \\
\hline 6 & Jubilant Life Sciences & 131 & 1 & 0 & 0 & 132 & 263 & 264 & 1.00 & 99.62 & 1 \\
\hline 7 & Mtd & 101 & 9 & 1 & 21 & 132 & 211 & 222 & 0.95 & 95.05 & 4 \\
\hline 8 & ONGC & 114 & 8 & 8 & 2 & 132 & 236 & 260 & 0.91 & 90.77 & 6 \\
\hline 9 & TCS & 126 & 3 & 3 & 0 & 132 & 255 & 264 & 0.97 & 96.59 & 3 \\
\hline 10 & Mahindra Rise & 115 & 4 & 11 & 2 & 132 & 234 & 260 & 0.90 & 90.00 & 7 \\
\hline 11 & ULTRATECH CEMENT & 125 & 0 & 7 & 0 & 132 & 250 & 264 & 0.95 & 94.70 & 5 \\
\hline
\end{tabular}




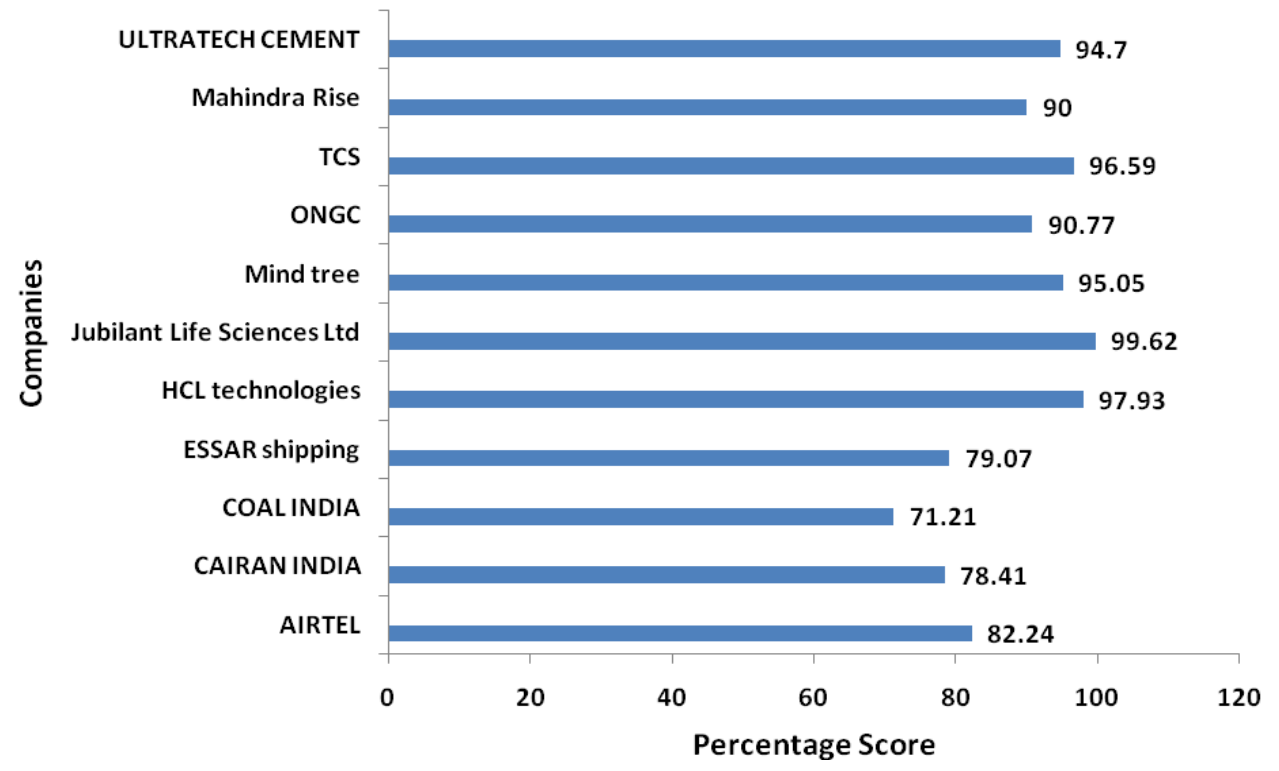

Figure 3: Company-wise disclosure for the year 2014-15 as per GRI G3.1

Table 6: Company-wise disclosure for the year 203-14 as per GRI G3.1

\begin{tabular}{|c|c|c|c|c|c|c|c|c|c|c|c|}
\hline \multicolumn{12}{|c|}{$\begin{array}{rrr}G 3.1 \\
2013-1\end{array}$} \\
\hline $\begin{array}{l}\text { Sr. } \\
\text { No. }\end{array}$ & COMPANY & $\mathbf{F}$ & $\mathbf{P}$ & No & NA & Total & $\mathbf{S}$ & $\mathbf{A S}$ & S/AS & Per & Rank \\
\hline 1 & $\mathrm{BP}$ & 105 & 17 & 10 & 0 & 132 & 227 & 264 & 0.86 & 85.98 & 19 \\
\hline 2 & CAIRAN INDIA & 99 & 10 & 23 & 0 & 132 & 208 & 264 & 0.79 & 78.79 & 23 \\
\hline 3 & COAL INDIA & 90 & 0 & 42 & 0 & 132 & 180 & 264 & 0.68 & 68.18 & 24 \\
\hline 4 & ESSAR shipping & 108 & 17 & 7 & 0 & 132 & 233 & 264 & 0.88 & 88.26 & 17 \\
\hline 5 & ESSAR ENERGY & 122 & 7 & 3 & 0 & 132 & 251 & 264 & 0.95 & 95.08 & 11 \\
\hline 6 & GAIL & 127 & 2 & 3 & 0 & 132 & 256 & 264 & 0.97 & 96.97 & 7.5 \\
\hline 7 & HDFC BANK & 100 & 8 & 20 & 4 & 132 & 208 & 256 & 0.81 & 81.25 & 22 \\
\hline 8 & HINDALCO & 100 & 28 & 4 & 0 & 132 & 228 & 264 & 0.86 & 86.36 & 18 \\
\hline 9 & $\mathrm{HP}$ & 120 & 8 & 4 & 0 & 132 & 248 & 264 & 0.94 & 93.94 & 13 \\
\hline 10 & JSW energy & 121 & 0 & 11 & 0 & 132 & 242 & 264 & 0.92 & 91.67 & 15 \\
\hline 11 & $\begin{array}{l}\text { Jubilant Life } \\
\text { Sciences Ltd }\end{array}$ & 131 & 1 & 0 & 0 & 132 & 263 & 264 & 1.00 & 99.62 & 5 \\
\hline 12 & $\begin{array}{l}\text { MAHINDRA } \\
\text { finance }\end{array}$ & 94 & 16 & 11 & 11 & 132 & 204 & 242 & 0.84 & 84.30 & 21 \\
\hline 13 & $\begin{array}{l}\text { MAHINDRA } \\
\text { LIFESPACE } \\
\text { DEVELOPERS } \\
\text { LIMITED }\end{array}$ & 128 & 1 & 2 & 1 & 132 & 257 & 262 & 0.98 & 98.09 & 6 \\
\hline 14 & Mind tree & 96 & 9 & 3 & 24 & 132 & 201 & 216 & 0.93 & 93.06 & 14 \\
\hline 15 & NTPC & 128 & 0 & 0 & 4 & 132 & 256 & 256 & 1.00 & 100.00 & 1 \\
\hline 16 & ONGC & 114 & 8 & 8 & 2 & 132 & 236 & 260 & 0.91 & 90.77 & 16 \\
\hline 17 & RELIANCE & 126 & 4 & 2 & 0 & 132 & 256 & 264 & 0.97 & 96.97 & 7.5 \\
\hline 18 & TATA MOTORS & 120 & 3 & 6 & 3 & 132 & 243 & 258 & 0.94 & 94.19 & 12 \\
\hline 19 & TATA POWER & 132 & 0 & 0 & 0 & 132 & 264 & 264 & 1.00 & 100.00 & 1 \\
\hline
\end{tabular}




\begin{tabular}{|c|l|c|c|c|c|c|c|c|c|c|c|}
\hline 20 & TATA STEEL & 132 & 0 & 0 & 0 & 132 & 264 & 264 & 1.00 & 100.00 & 1 \\
\hline 21 & TCS & 132 & 0 & 0 & 0 & 132 & 264 & 264 & 1.00 & 100.00 & 1 \\
\hline 22 & Mahindra Rise & 111 & 5 & 16 & 0 & 132 & 227 & 264 & 0.86 & 85.98 & 20 \\
\hline 23 & Tech Mahindra & 114 & 7 & 1 & 10 & 132 & 235 & 244 & 0.96 & 96.31 & 9 \\
\hline 24 & $\begin{array}{l}\text { ULTRATECH } \\
\text { CEMENT }\end{array}$ & 127 & 0 & 5 & 0 & 132 & 254 & 264 & 0.96 & 96.21 & 10 \\
\hline
\end{tabular}

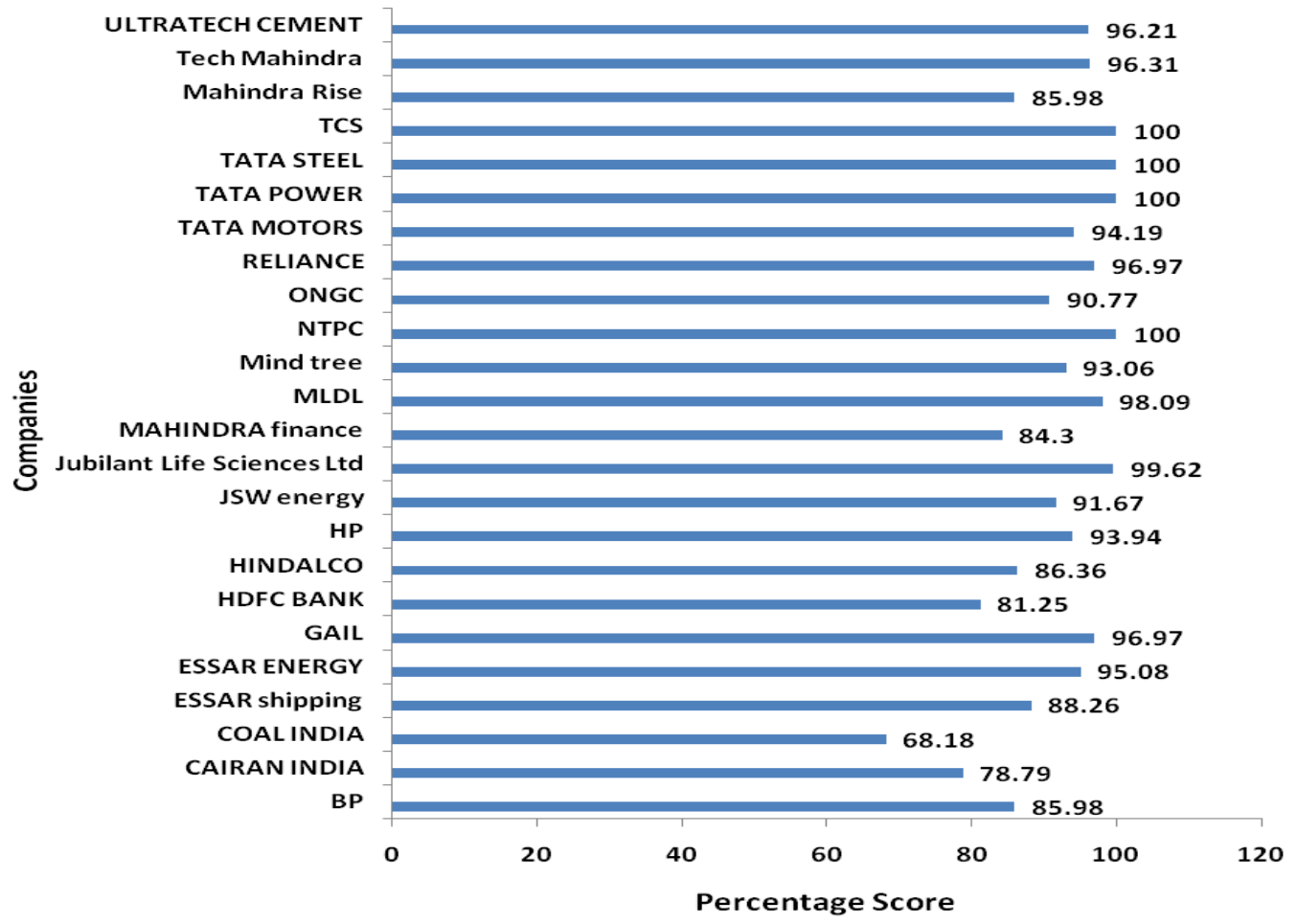

Figure 4: Company-wise disclosure for the year 2013-14 as per GRI G3.1

The following observations are obtained from the above table:

Table 7: Observations obtained

\begin{tabular}{|c|c|c|c|}
\hline \multirow{2}{*}{ GRI } & Year & Minimum percent score & Maximum percent score \\
\hline \multirow{2}{*}{ G4 } & $2014-15$ & 34.00 (SAIL) & 99.33 (IOCL) \\
\cline { 2 - 4 } & $2013-15$ & $\begin{array}{c}26.67 \text { (Godrej \& Boyce } \\
\text { Manufacturing) }\end{array}$ & 96.00 (IOCL) \\
\hline \multirow{2}{*}{ G 3.1 } & $2014-15$ & 71.21 (COAL INDIA) & 99.62 (Jubilant Life Sciences Ltd) \\
\cline { 2 - 4 } & $2013-15$ & 68.18 (COAL INDIA) & 100 (TCS, TATA STEEL, TATA POWER, \\
\end{tabular}

An increase has been noticed in the percentage score of companies in both the versions. More companies have started reporting on sustainability measures. Companies opt the latest version of GRI for reporting on sustainability.

Industry-Wise Disclosure

To study the industry wise sustainability disclosure practices of Indian companies; the companies reporting on GRI G4 for the year 2014-15 were grouped industry wise. Percentage mean scores were 
obtained to study industry wise sustainability disclosure practices. This has been shown in Table below:

Table 8: Industry-wise disclosure for the year 2014-15 as per GRI G4

\begin{tabular}{|c|c|c|c|c|c|}
\hline \multicolumn{6}{|c|}{ Industry-wise Disclosure Scores } \\
\hline \multicolumn{6}{|c|}{$\begin{array}{c}\text { G4 } \\
2014-15 \\
\end{array}$} \\
\hline $\begin{array}{l}\text { SR } \\
\text { NO }\end{array}$ & COMPANY & Sector & $\begin{array}{l}\text { Industry } \\
\text { Group }\end{array}$ & $\begin{array}{l}\text { Percentage } \\
\text { Score }\end{array}$ & $\begin{array}{l}\text { Percentage } \\
\text { Mean Score }\end{array}$ \\
\hline 6 & HONDA & $\begin{array}{l}\text { AUTO - } 2 \& 3 \\
\text { WHEELERS }\end{array}$ & \multirow{2}{*}{ Automobile } & 81.25 & \multirow{2}{*}{71.50} \\
\hline 19 & TATA MOTORS & Auto - LCVs \& HCVs & & 61.74 & \\
\hline 1 & AXIS BANK & Banks - Private Sector & \multirow{4}{*}{ Finance } & 72.00 & \multirow{4}{*}{62.31} \\
\hline 4 & HDFC BANK & Banks - Private Sector & & 63.64 & \\
\hline 23 & YES BANK & Banks - Private Sector & & 67.33 & \\
\hline 14 & $\begin{array}{l}\text { MAHINDRA } \\
\text { finance }\end{array}$ & $\begin{array}{l}\text { FINANCE - LEASING \& } \\
\text { HIRE PURCHASE }\end{array}$ & & 46.26 & \\
\hline 8 & INFOSYS & Computers - Software & \multirow{2}{*}{ IT } & 71.58 & \multirow{2}{*}{68.45} \\
\hline 22 & Tech Mahindra & Computers - Software & & 65.33 & \\
\hline 3 & GAIL & \begin{tabular}{|l} 
OIL DRILLING AND \\
EXPLORATION \\
\end{tabular} & \multirow{2}{*}{$\begin{array}{c}\text { OIL } \\
\text { DRILLING } \\
\text { AND } \\
\text { EXPLORATI } \\
\text { ON } \\
\end{array}$} & 86.58 & \multirow[b]{2}{*}{68.29} \\
\hline 16 & OIL INDIA & $\begin{array}{l}\text { OIL DRILLING AND } \\
\text { EXPLORATION }\end{array}$ & & 50.00 & \\
\hline 2 & $\mathrm{BP}$ & REFINERIES & \multirow{3}{*}{ REFINERIES } & 92.47 & \multirow{3}{*}{77.04} \\
\hline 7 & HP & REFINERIES & & 39.33 & \\
\hline 9 & IOCL & REFINERIES & & 99.33 & \\
\hline 17 & SAIL & STEEL - LARGE & \multirow{3}{*}{ STEEL } & 34.00 & \multirow{3}{*}{47.27} \\
\hline 21 & TATA STEEL & STEEL - LARGE & & 73.15 & \\
\hline 11 & \begin{tabular}{|l} 
Jindal Steel \& \\
Power Ltd JSPL
\end{tabular} & STEEL - SPONGE IRON & & 34.67 & \\
\hline 5 & HINDALCO & Aluminium & \multirow{7}{*}{ Others } & 92.33 & \multirow{7}{*}{77.08} \\
\hline 12 & JK CEMENT & CEMENT - MAJOR & & 34.67 & \\
\hline 18 & $\begin{array}{l}\text { TATA } \\
\text { CHEMICALS }\end{array}$ & Chemicals & & 65.33 & \\
\hline 10 & ITC & CIGARETTES & & 85.86 & \\
\hline 15 & NEROLAC & PAINTS \& VARNISHES & & 85.33 & \\
\hline 20 & TATA POWER & $\begin{array}{l}\text { POWER - GENERATION } \\
\text { \& DISTRIBUTION }\end{array}$ & & 98.67 & \\
\hline 13 & L\&T & $\begin{array}{l}\text { INFRASTRUCTURE - } \\
\text { GENERAL }\end{array}$ & & 77.33 & \\
\hline
\end{tabular}




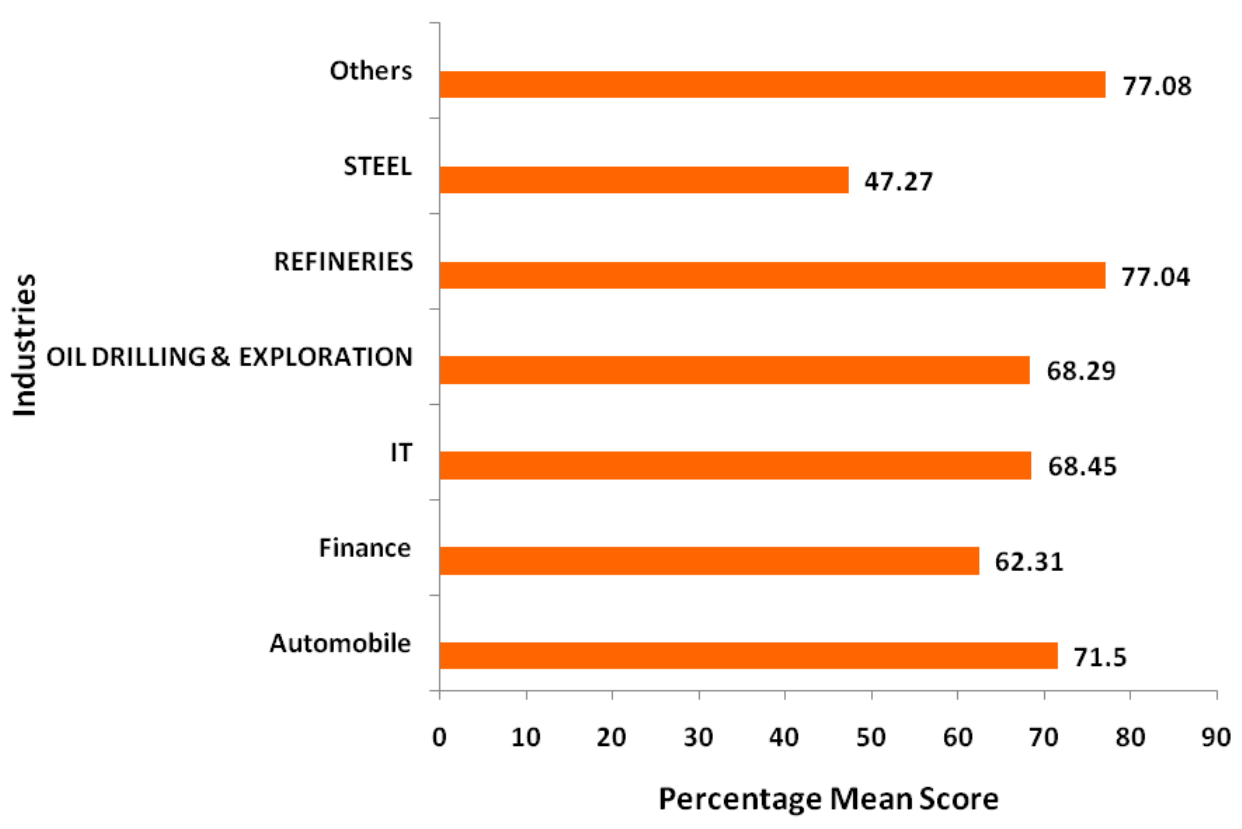

Figure 5: Industry-wise disclosure for the year 2014-15 as per GRI G4

From the above table it is observed that: the industry score ranges from $47.27 \%$ (Steel sector) to $77.08 \%$ (other sectors). However, except steel sector almost all sectors score above average.

Further, industry wise disclosures were analysed using one way ANOVA to see if the disclosure practices of companies among industries differ significantly from each other or not. For this purpose the disclosures of companies chosen was for the year 2014-15 and reporting as per GRI G4. The following table shows the results of ANOVA:

Table 9: ANOVA

INDPERSC

\begin{tabular}{|l|r|r|r|l|l|}
\hline & \multicolumn{1}{|c|}{$\begin{array}{c}\text { Sum of } \\
\text { Squares }\end{array}$} & df & Mean Square & F & Sig. \\
\hline Between Groups & 3679.990 & 11 & 334.545 & 8.731 & .259 \\
Within Groups & 38.316 & 1 & 38.316 & & \\
Total & 3718.307 & 12 & & & \\
\hline
\end{tabular}

It can be observed from the Table 5 that the value of $\mathrm{F}(8.731)$ is not significant at the $5 \%$ percent level. Thus, no statistically significant variation has been found in the mean disclosure scores of various industry groups. Therefore, the null hypothesis H0 has been accepted that there is no significant difference in the inter industry disclosure scores. This shows that Indian companies do not give much consideration to their industries characteristics while disclosing information in their sustainability reports.

\section{Conclusions:}

The major finding of the study can be summarized as follows:

$>$ Indian companies have recognized the importance of sustainability reporting and the major companies are reporting on it.

$>$ More companies have started using the latest version of GRI for reporting on sustainability.

$>$ An increase has been noticed in the percentage score of companies in both the versions of GRI (G3.1 \& G4).

$>$ Indian companies do not give much consideration to their industries characteristics while disclosing information in their sustainability reports. 


\section{References:}

Bhalla, R., \& Bansal, S. K. (2014). Corporate Sustainability Reporting: A Study of Economic Sustainability Aspect by Selected Indian Corporations. International jounal of current research and academic review , 2 (5), 37-46.

Bhatia, A., \& Tuli, S. (2014). Sustainable Disclosure Practices:A Study of Sensex Companies in India. Indian Journal of Corporate Governance , 7 (1), 39-55.

Daizy, \& Das, N. (2014). Sustainability reporting framework: comparative analysis of global reporting initiatives and dow jones sustainability index. International Journal of Science, Environment and Technology, , 3 (1), 55 - 66.

Godha, A., \& Jain, P. (2015). Sustainability Reporting Trend in Indian Companies as per GRI Framework:A Comparative Study. South Asian Journal of Business and Management Cases , 4 (1), $62-73$.

Goyal, N. (2014). Corporate Sustainability Reporting Practices among Indian Companies - Myth or Reality. International Journal of Management and Social Sciences Research , 3 (1), 54-60.

Kumar, R. (2014). A study on sustainability reporting practices in indian and global companies with special references to the petroleum companies. Abhinav National Monthly Refereed Journal of Research in commerce and managment , 3 (4), 15-25.

Mitra, P. K. (2012). Sustainability reporting practices in India: its problems and prospects. International journal of marketing, financial services and mnagement research , l (5), 109-115. 